\title{
Brugada syndrome masked by complete left bundle branch block: A clinical and functional study of its association with the p.1449Y>H SCN5A variant
}

\author{
Eduardo Arana-Rueda MD, PhD ${ }^{1,2}$ () | María R. Pezzotti PhD ${ }^{2}$ | \\ Alonso Pedrote MD, $\mathrm{PhD}^{1}$ (1) | Juan Acosta MD, $\mathrm{PhD}^{1}$ | Manuel Frutos-López MD \\ Lourdes-María Varela PhD ${ }^{2}$ | Noelia García-Fernández ${ }^{2}$ | Antonio Castellano $\mathrm{PhD}^{2,3}$ (1)
}

\author{
${ }^{1}$ Department of Cardiology, Arrhythmia Unit, \\ Virgen del Rocío University Hospital, \\ Sevilla, Spain \\ ${ }^{2}$ Departamento de Fisiología Médica y \\ Biofísica, Instituto de Biomedicina de Sevilla \\ (IBiS)/Hospital Universitario Virgen del Rocío/ \\ CSIC, Universidad de Sevilla, Sevilla, Spain \\ ${ }^{3}$ CIBERCV, ISCIII, Madrid, Spain

\section{Correspondence} \\ Eduardo Arana-Rueda, MD, PhD, Department \\ of Cardiology, Arrhythmia Unit, Virgen del \\ Rocío University Hospital, Avda. Manuel \\ Siurot, s/n; 41013 Sevilla, Spain. \\ Email: eduardo.arana.sspa@juntadeandalucia.es \\ Antonio Castellano, PhD, Instituto de \\ Biomedicina de Sevilla (IBiS), Virgen del Rocío \\ University Hospital, Avda. Manuel Siurot, s/n; \\ 41013 Sevilla, Spain. \\ Email: acastell@us.es \\ Funding information \\ Sociedad Española de Cardiología: Ritmo \\ 2017; Consejería de Salud y Familias, Junta de \\ Andalucía, Grant/Award Number: \\ PI-0365-2017
}

\begin{abstract}
SCN5A gene variants are associated with both Brugada syndrome and conduction disturbances, sometimes expressing an overlapping phenotype. Functional consequences of SCN5A variants assessed by patch-clamp electrophysiology are particularly beneficial for correct pathogenic classification and are related to disease penetrance and severity. Here, we identify a novel SCN5A loss of function variant, p.1449Y $>\mathrm{H}$, which presented with high penetrance and complete left bundle branch block, totally masking the typical findings on the electrocardiogram. We highlight the possibility of this overlap combination that makes impossible an electrocardiographic diagnosis and, through a functional analysis, associate the $\mathrm{p} .1449 \mathrm{Y}>\mathrm{H}$ variant to SCN5A pathogenicity.
\end{abstract}

\section{KEYWORDS}

Brugada syndrome, left bundle branch block, loss of function, patch clamp, SCN5A

\section{1 | INTRODUCTION}

To date, the diagnosis of Brugada syndrome (BrS) is still based on the correct identification of the typical electrocardiogram (ECG) pattern. ${ }^{1}$ Of more than $20 \mathrm{BrS}$-associated genes, only some variants in SCN5A gene show definitive evidence of its association with $\mathrm{BrS}$ phenotype. ${ }^{2}$ These SCN5A variants, affecting sodium channel function, can also be linked to conduction disturbances, sometimes combining in the same patient. ${ }^{3}$

In this report, we described a patient with definitive diagnosis of $\mathrm{BrS}$ and transient complete left bundle branch block (CLBBB) that totally masked the typical ECG pattern and establish its causal relationship with a new variant in SCN5A by means of a functional study. 


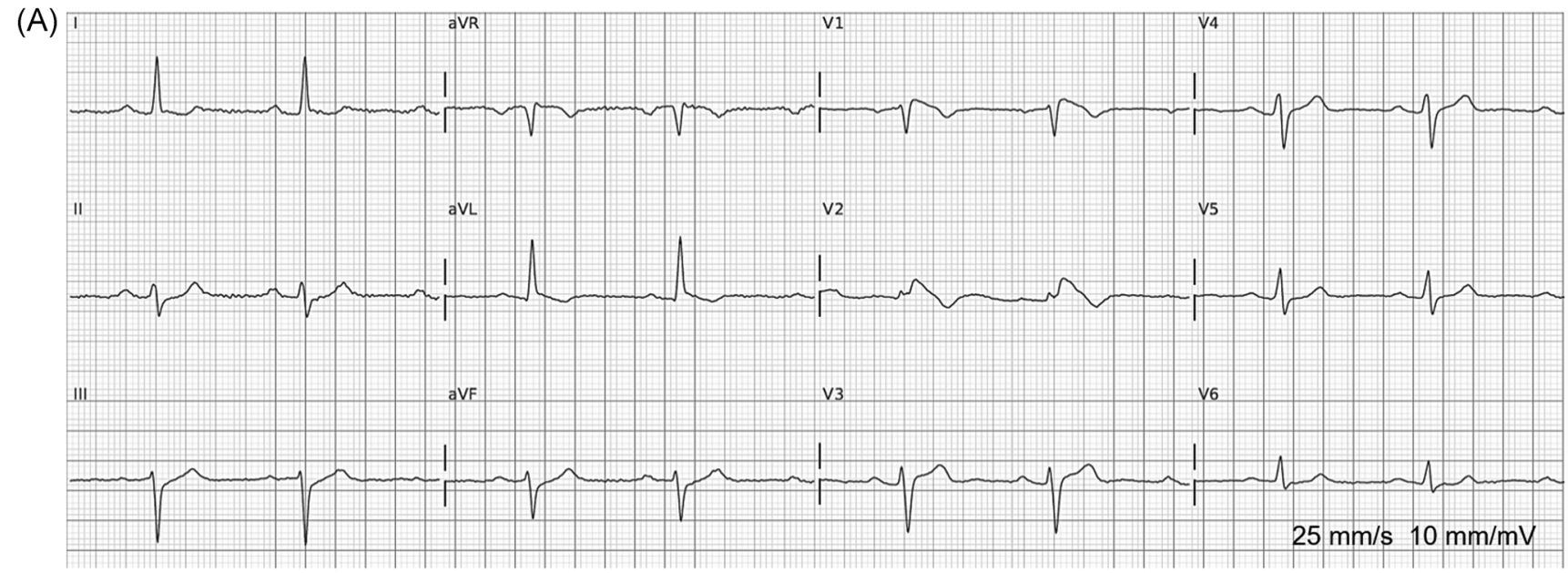

(B)

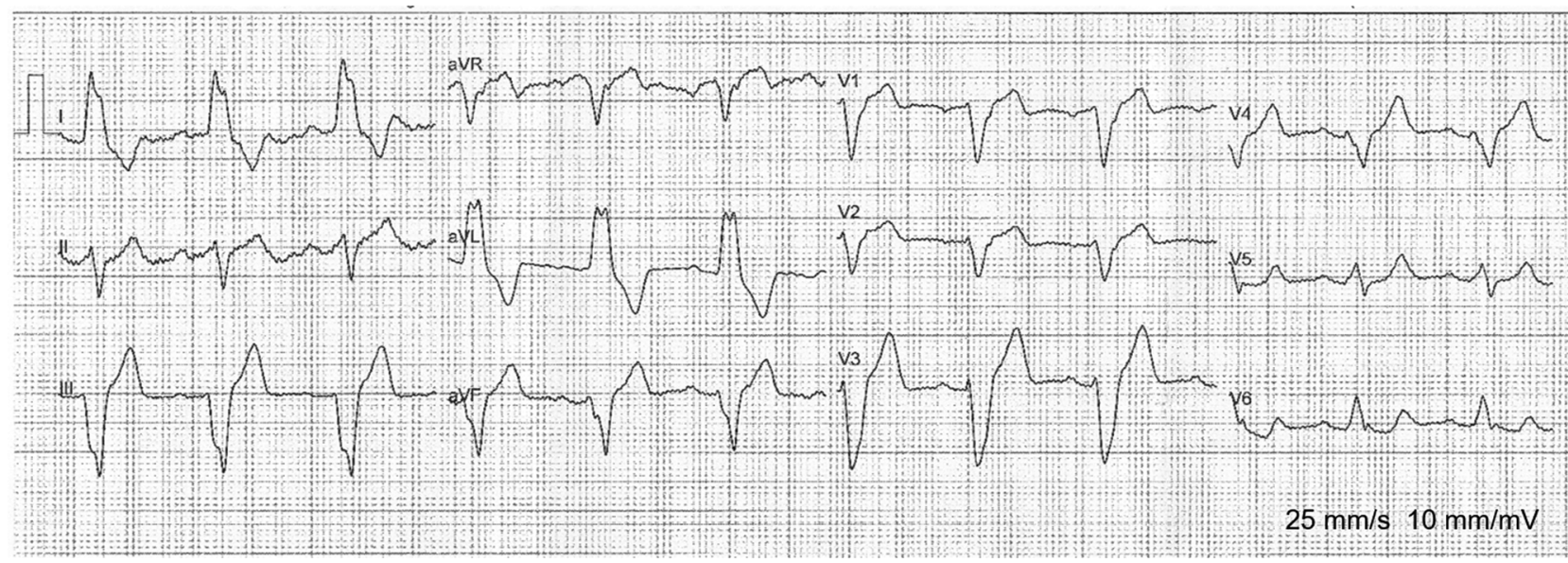

FIGURE 1 (A) ECG recording obtained at initial diagnosis, displaying typical coved Brugada pattern in V1 and V2 (fourth intercostal space). It has sinus rhythm at $60 \mathrm{bpm}$ and has signs of altered conduction with a PR interval of $220 \mathrm{~ms}$ and a left anterior cardiac hemiblock. (B) ECG tracing obtained 5 years later in a routine checking. It shows sinus rhythm at $71 \mathrm{bpm}$ with CLBBB, totally obscuring the repolarization characteristics of Brugada pattern. CLBBB, complete left bundle branch block; ECG, electrocardiogram

\section{1 | Case presentation}

A 48-years-old lady was referred to our center because of anomalous ECG recording during a medical evaluation for palpitations. It was concordant with persistent type-1 BrS pattern, with firstdegree heart block and left axis deviation (Figure 1A). She had no family history of sudden death, significant medical history, or others symptoms previous to the episode and was not under any medical treatment. Structural heart diseases were ruled out. After obtaining written consent, we conducted an electrophysiologic study which revealed a His-Purkinje interval of $60 \mathrm{~ms}$. Programmed atrial and ventricular stimulation with up to 3 extrastimuli in right ventricular apex was not able to induce arrhythmias. A next-generation genetic test was performed, leading us to identify the heterozygous variant c.4345T>C (see below), in exon 25 of the SCN5A gene, classified as a variant of unknown significance (VUS). The patient was advised to make lifestyle changes for the prevention of arrhythmias and followed up in our outpatient clinic. Five years later, in a routine checking, her ECG dramatically changed showing CLBBB (Figure 1B). A new evaluation was done with no appearance of structural cardiac disease. A $24 \mathrm{~h}$ ECG Holter revealed an intermittent and rate-dependent CLBBB. A new electrophysiological study showed an HV interval of $75 \mathrm{~ms}$, with Phase 3 aberrancy registered spontaneously with subtle accelerations of sinus rhythm and with atrial stimulation (Figure 2A,B). No arrhythmias were induced. Four years later, the patient remains with no cardiac events in a close follow-up.

A familial screening was done, identifying a resting ECG consistent with type-2 Brugada pattern with prominent sinus bradycardia in one asymptomatic son (24 years old). A flecainide test revealed a type-1 Brugada ECG pattern. This patient was the only carrier of the SCN5A variant in the family and also the only one with BrS phenotype on ECG. Reviewing our series of patients with $\mathrm{BrS}$, this variant was also present in three members $(18,45$, and 46 years old) of two different families. All these cases had spontaneous type-1 ECG pattern but without associated conduction anomalies nor symptoms.

The c.4345T >C variant (Figure 2C) induces the substitution of the conserved tyrosine 1449 by a histidine (p.1449Y $>\mathrm{H}$; Figures 2D and S1). In silico analysis tools (Polyphen2, Mutation Taster, Provean and REVEL) were used to clarify the significance of the SCN5A 
(A)

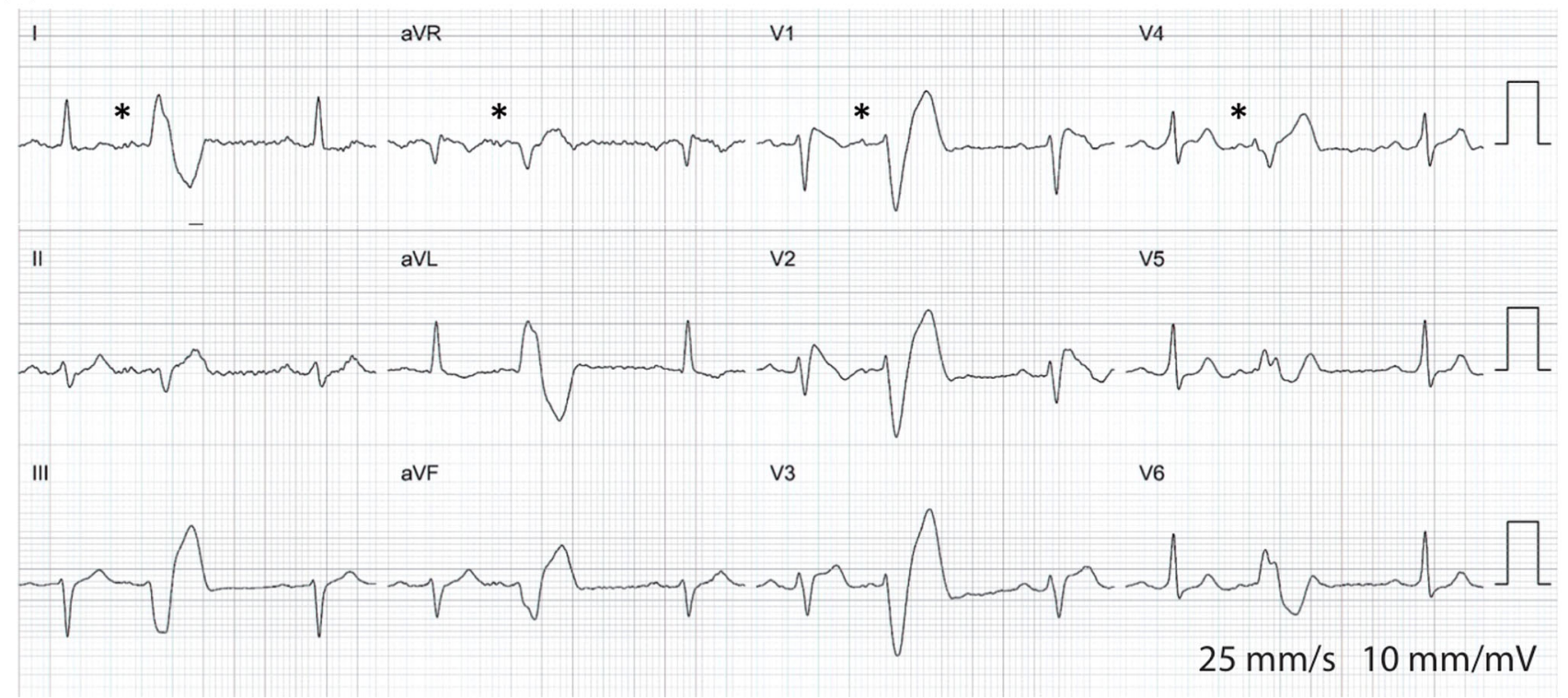

(B)

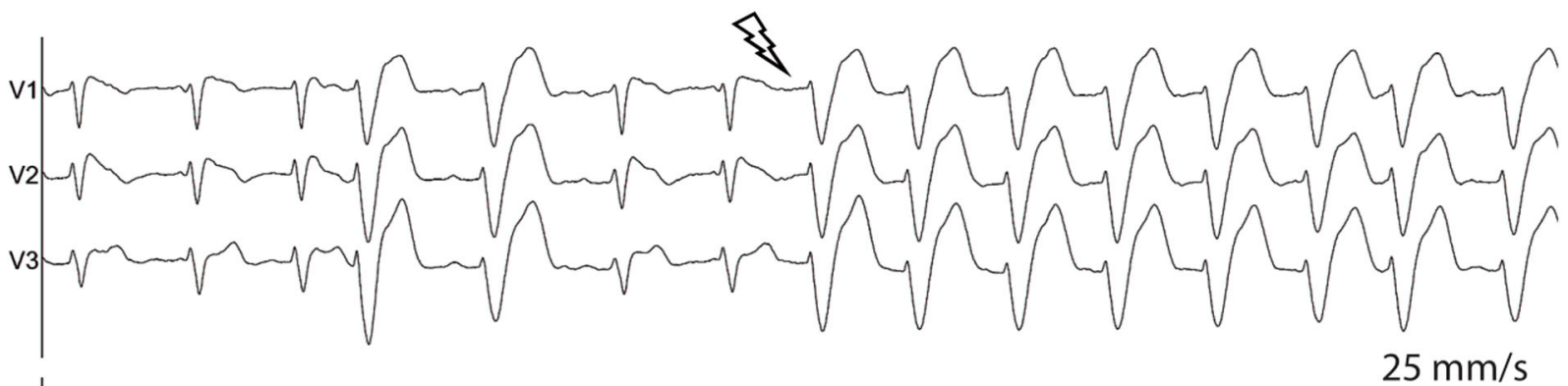

(C)

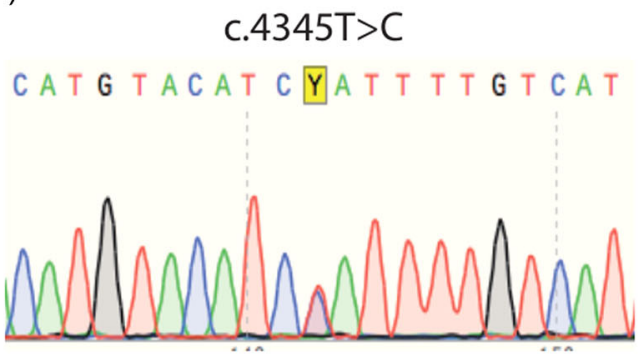

(D)

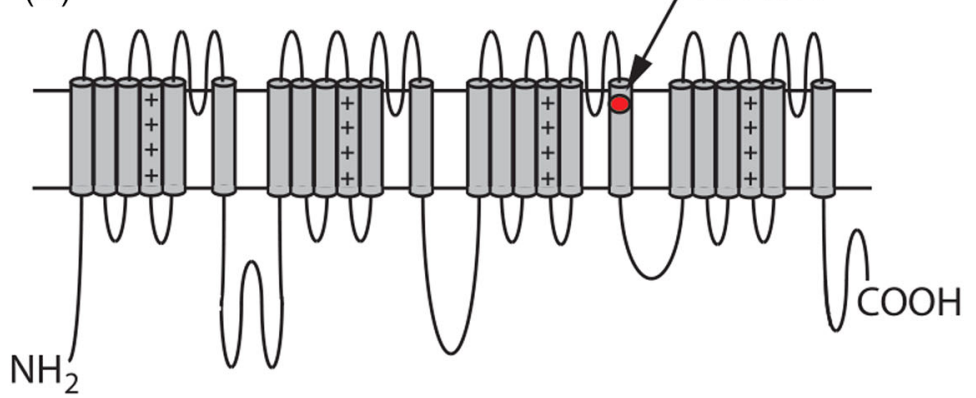

FIGURE 2 (A) ECG recording showing Phase 3 aberration in premature atrial depolarizations (*), obscuring the typical Brugada pattern. The same phenomenon is shown with atrial stimulation (ray) during the electrophysiological study over a basal junctional rhythm (B). (C) DNA sequence chromatogram depicting the heterozygous variation (c.4345T $>C$ ) of the SCN5A gene leading to the missense variant $\mathrm{p} .1449 \mathrm{Y}>\mathrm{H}$. (D) Scheme of the $\mathrm{Na}_{\mathrm{v}} 1.5$ channel showing the location of the $\mathrm{p} .1449 \mathrm{Y}>\mathrm{H}(\mathrm{Y} 1449 \mathrm{H})$ variant. ECG, electrocardiogram

p.1449Y $>\mathrm{H}$ variant, and with the exception of Polyphen2 (score 0.320 ), the rest of them supported a deleterious effect for this variant.

\section{2 | SCN5A p.1449Y>H functional study}

To better assess the relevance of the detected variant we investigated whether alterations in the biophysical properties of the
p.1449Y $>\mathrm{H}$ mutant could explain the observed phenotype. Sodium currents were recorded in patch-clamp experiments using HEK293 cells $24-48 \mathrm{~h}$ after transfection with wild-type (WT) or p.1449Y $>\mathrm{H}$ $\mathrm{Na}_{\mathrm{v}} 1.5$ channels, together with the human $\beta_{1}$ subunit. The p.1449Y $>\mathrm{H}$ mutant generated very little, but detectable, current $(-7.91 \pm 0.75 \mathrm{pA} / \mathrm{pF})$, about $2 \%$ of the current density generated by the WT channel $(409 \pm 52 \mathrm{pA} / \mathrm{pF}$; Figure $3 \mathrm{~A}, \mathrm{~B})$. This result suggested that the mutant channel reaches the plasma membrane but is not 

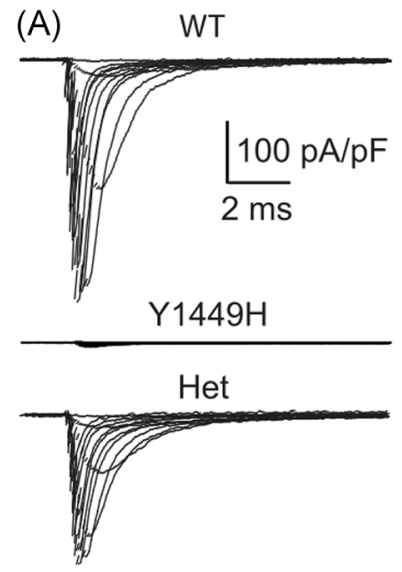

(C)

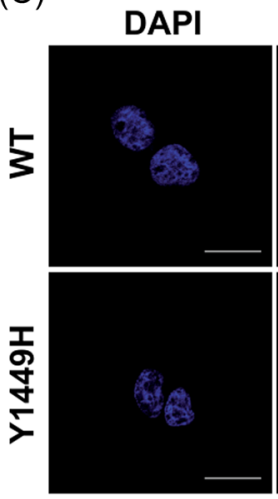

(B)

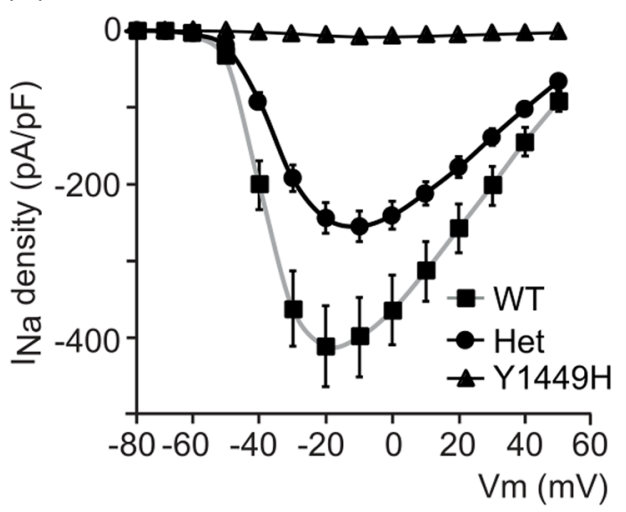

FIGURE 3 Functional analysis of the p.1449Y $>\mathrm{H}$ mutant. (A) Representative current traces obtained from HEK293 cells transfected with $\mathrm{Na}_{\mathrm{v}} 1.5 \mathrm{WT}$, p.1449Y>H (Y1449H), or WT + p.1449Y>H (Het) channels and the $\mathrm{Na}_{\mathrm{v}} \mathrm{\beta 1}$ subunit. (B) Current density-voltage relationships in the same groups of cells. (C) Representative confocal images from HEK293 cells transfected with the FLAG-tagged $\mathrm{Na}_{\mathrm{v}} 1.5 \mathrm{WT}$ and p.1449Y > H constructs. Scale bar: $20 \mu \mathrm{m}$ fully functional. Our SCN5A constructs include an extracellular FLAG tag, located in the domain I (DI) S1-S2 linker of the $\mathrm{Na}_{\mathrm{v}} 1.5$ protein. ${ }^{4}$ To analyze whether p.1449Y>H mutant traffics to the plasma membrane, anti-FLAG immunofluorescence was performed using non-permeabilized cells. Confocal microscopy analysis revealed that the mutant channel reaches the plasma membrane (Figure $3 \mathrm{C}$ ), confirming that the variant does not significantly alter channel trafficking.

Several reports have described $\mathrm{BrS}$ variants displaying dominantnegative effects, ${ }^{5,6}$ as a result of the dimerization and coupled gating of the WT and mutant $\mathrm{Na}^{+}$channel $\alpha$-subunits. ${ }^{6}$ Thus, and mimicking the heterozygous condition (Het), we tested whether p.1449Y>H variant could exert any dominant-negative effect on WT $\mathrm{Na}_{v} 1.5$ channel. When WT and p.1449Y>H channels were co-expressed (in a 1:1 ratio), sodium channel currents were recorded, with a peak current density of $253 \pm 20 \mathrm{pA} / \mathrm{pF}$, approximately $60 \%$ of that observed in the cells transfected with the WT channel (Figure $3 A, B)$. Further comparison of the WT and Het channels biophysical properties discarded any major dominant-negative effect of the p.1449Y $>\mathrm{H}$ variant (see Figure S2 and Table S1).

\section{2 | DISCUSSION}

This article shows how patients with BrS can develop a CLBBB totally masking the typical ECG pattern, and highlights the awareness that must be taken for a correct diagnosis if both conditions are present.
Through a clinical and functional study, we demonstrate how the p. $1449 Y>H$ SCN5A variant causes a significant loss of function in sodium channel that may be associated with this overlapping phenotype.

$\mathrm{BrS}$ is an inherited disorder associated with sudden death along with the signature of a characteristic ECG pattern in precordial leads. Thus, the current clinical diagnosis of $\mathrm{BrS}$ is based on the demonstration of a typical ECG pattern either spontaneous or after IC pharmacological challenge ${ }^{1}$ (Figure 1A). Nowadays, these criteria are not replaceable by any other diagnostic method. However, this finding can be clinically difficult, as the pattern is characterized by its dynamic behavior and the association with various and often extensive conduction diseases. The SCN5A gene, which encodes the cardiac sodium channel a subunit $\mathrm{Na}_{\mathrm{v}} 1.5$, is the most common $\mathrm{BrS}$-associated gene, but is found in only $20 \%-25 \%$ of probands. ${ }^{1}$ This channel is responsible for excitability and impulse conduction in the contractile myocardium and specialized conduction system, and is also implicated in refractoriness and repolarization. Thus, loss of function variants in SCN5A have been associated not only with BrS, but also with early repolarization syndrome, a variety of conduction diseases and overlapping syndrome. ${ }^{1,3}$ Indeed, there is a significant clinical and genetic overlap between $\mathrm{BrS}$ and progressive cardiac conduction disease, and both conditions may coexist or manifest in isolated forms in carriers of the same variant within the same family. ${ }^{3}$ Maury et al., ${ }^{7}$ studied the prevalence of conduction disturbances in patients with $\mathrm{BrS}$ and reported a high proportion of complete right bundle branch block (CRBBB). In such cases, even experienced electrophysiologists may have difficulties in 
discriminating between BrS and CRBBB by ECG alone. Nevertheless, the prevalence of other intraventricular conduction abnormalities in $\mathrm{BrS}$ patients is less known, with CLBBB estimation of less than $1 \%$. In CLBBB the ventricles are activated sequentially, first the right ventricle via the right bundle and then the left ventricle, slowly activated through the septum with forces directed towards the left, posteriorly, and inferiorly. As a result, the ECG changes indicative of $\mathrm{BrS}$ are not seen in precordial leads, as they are buried within a wider QRS complex due to delayed activation of the left ventricle. Our case, with a CLBBB with Phase 3 characteristics developed during follow-up, is a clear example of this combination and illustrates the total concealing of the ECG pattern, making impossible a $\mathrm{BrS}$ diagnosis in cases of persistent CLBBB. In such cases, high clinical suspicion, based on age at presentation, associated disorders, symptomatology, and family history, is necessary for ruling out this rare association. The role of specific pacing maneuvers to unmask the BrS pattern under persistent CLBBB, as occurs in concealed $\mathrm{BrS}$ due to $\mathrm{CRBBB}$, remains to be explored.

At this time, there is a general concern about the pathogenicity of many variants in SCN5A previously identified as implicated in $\mathrm{BrS}$, but of ambiguous significance following the current guidelines for classification (up to $63 \%$ VUS). ${ }^{2,7}$ Nowadays, functional evidence is considered the major score driver for pathogenicity assumption for missense variants. According to a recent re-evaluation of the American College of Medical Genetics and Genomics and the Association for Molecular Pathology (ACMG-AMP) rules for assessment of pathogenicity, ${ }^{2,9}$ the SCN5A variant identified in this report can be classified as pathogenic, since it fulfills the following criteria: (i) the $\mathrm{p} .1449 \mathrm{Y}>\mathrm{H}$ variant is a missense variant that leads to the replacement of tyrosine by histidine at position 1449, in the transmembrane S6 region of DIII, which is part of the pore region of the $\mathrm{Na}_{v} 1.5$ channel and, according to the SCN5A variant browser, is a hotspot region for BrS1 (ACMG category PM1, moderate evidence); ${ }^{8}$ (ii) other variants producing amino acid changes in the same residue has been previously established as pathogenic and associated to clinical $\mathrm{BrS}$ ( $\mathrm{p} .1449 \mathrm{Y}>\mathrm{S}$ ) and also to conduction disease with partial loss of function in in vitro studies (p.1449Y>C; PS1, strong evidence). ${ }^{9-12}$ These results indicate that the conserved $Y 1449$ is crucial for the proper functioning of the $\mathrm{Na}_{\mathrm{v}} 1.5$ channel, and that its alteration induces dramatic changes in channel activity; and (iii) p. $1449 \mathrm{Y}>\mathrm{H}$ mutant encodes a not fully functional channel that generates extremely small ( $2 \%$ as compared to the WT) sodium currents, and a decrease more than $50 \%$ in the peak current is significantly associated with $\mathrm{BrS1}$ penetrance (PS3, strong evidence). ${ }^{2,9}$

Recently, Ciconte et al., ${ }^{13}$ demonstrated that BrS carriers of SCN5A pathogenic variants exhibit a more aggressive clinical presentation and a greater epicardial substrate on electrophysiological studies, associating genotype with phenotypic expression. Although p.1449 $\mathrm{Y}>\mathrm{H}$ confers a high penetrance in the cases analyzed in this study, no one has symptoms of severity during close follow-up. However, a hypothetical more serious phenotype could be developed with aging, since some experimental studies have demonstrated the effect of aging and myocardial fibrosis on the decline of expression and function of sodium channels. ${ }^{3}$ Special awareness should be given to these patients, monitoring the possible progression of the disease (symptoms, periodical ECG, and Holter evaluation) and periodically reassessing the risk of sudden death in a multiparametric manner.

\section{3 | CONCLUSIONS}

The combination of BrS and CLBBB as part of an overlap syndrome totally masks the typical BrS ECG pattern needed for diagnosis. We demonstrate how the $\mathrm{p} .1449 \mathrm{Y}>\mathrm{H}$ SCN5A variant produces a significant loss of function in sodium channel which is manifested as a marked phenotypical expression.

\section{ACKNOWLEDGMENTS}

We would like to thank Drs. Sara Pagans and Ramón Brugada for the kind gift of the pcDNA3-SCN5A plasmid containing the $\mathrm{hNa}$ 1.5 sequence, and Dr. Eva Delpón for the $\mathrm{hNav} \beta_{1} \mathrm{cDNA}$. This study was supported by grants from the Sociedad Española de Cardiología (Ritmo 2017), and the Consejería de Salud of the Junta de Andalucía (PI-0365-2017).

\section{CONFLICT OF INTERESTS}

The authors declare that there are no conflict of interests.

\section{DATA AVAILABILITY STATEMENT}

The data that support the findings of this study are available on request from the corresponding author. The data are not publicly available due to privacy or ethical restrictions.

\section{ORCID}

Eduardo Arana-Rueda (D) http://orcid.org/0000-0001-8132-7045

Alonso Pedrote (D) http://orcid.org/0000-0003-1677-2956

Antonio Castellano (D) http://orcid.org/0000-0003-3955-5137

\section{REFERENCES}

1. Antzelevitch C, Yan G-X, Ackerman MJ, et al. J-Wave syndromes expert consensus conference report: emerging concepts and gaps in knowledge. Europace. 2017;19:665-694.

2. Denham NC, Pearman CM, Ding WY, et al. Systematic re-evaluation of SCN5A variants associated with Brugada syndrome. J Cardiovasc Electrophysiol. 2019;30:118-127.

3. Asatryan B, Medeiros-Domingo A. Molecular and genetic insights into progressive cardiac conduction disease. Europace. 2019;21:1145-1158.

4. Wu G, Ai T, Kim JJ, et al. alpha-1-syntrophin mutation and the longQT syndrome: a disease of sodium channel disruption. Circ Arrhythm Electrophysiol. 2008;1:193-201.

5. Keller DI, Rougier JS, Kucera JP, et al. Brugada syndrome and fever: genetic and molecular characterization of patients carrying SCN5A mutations. Cardiovasc Res. 2005;67:510-519.

6. Clatot J, Ziyadeh-Isleem A, Maugenre S, et al. Dominant-negative effect of SCN5A N-terminal mutations through the interaction of $\mathrm{Na}_{v} 1.5$ a-subunits. Cardiovasc Res. 2012;96:53-63.

7. Richards S, Aziz N, Bale S, et al. Standards and guidelines for the interpretation of sequence variants: a joint consensus recommendation of the American College of Medical Genetics and Genomics and the Association for Molecular Pathology. Genet Med. 2015;17:405-424.

8. Kroncke BM, Glazer AM, Smith DK, Blume JD, Roden DM. SCN5A $\left(\mathrm{Na}_{\mathrm{v}} 1.5\right)$ variant functional perturbation and clinical presentation: variants of a certain significance. Circ Genomic Precis Med. 2018;11(5):e002095. 
9. Kapplinger JD, Tester DJ, Alders M, et al. An international compendium of mutations in the SCN5A-encoded cardiac sodium channel in patients referred for Brugada syndrome genetic testing. Heart Rhythm. 2010;7:33-46.

10. Jiménez-Jáimez J, Álvarez M, Algarra M, et al. Low clinical penetrance in causal mutation carriers for cardiac channelopathies. Rev Española Cardiol. 2013;66:275-281.

11. Hothi SS, Ara F, Timperley JP. Y1449C SCN5A mutation associated with overlap disorder comprising conduction disease, Brugada syndrome, and atrial flutter. J Cardiovasc Electrophysiol. 2015;26:93-97.

12. Glazer AM, Wada $\mathrm{Y}$, Li B, et al. High-throughput reclassification of SCN5A variants. Am J Hum Genet. 2020;107:111-123.

13. Ciconte G, Monasky MM, Santinelli V, et al. Brugada syndrome genetics is associated with phenotype severity. Eur Heart J. 2021; 42(11):1082-1090.

\section{SUPPORTING INFORMATION}

Additional Supporting Information may be found online in the supporting information tab for this article.

How to cite this article: Arana-Rueda E, Pezzotti MR, Pedrote $A$, et al. Brugada syndrome masked by complete left bundle branch block: A clinical and functional study of its association with the p.1449Y $>\mathrm{H}$ SCN5A variant. J Cardiovasc Electrophysiol. 2021;32:2785-2790.

https://doi.org/10.1111/jce.15215 